\title{
New results of the OPERA long-baseline experiment in the CNGS neutrino beam
}

\author{
Maximiliano Sioli* \\ Bologna University \& INFN \\ E-mail: sioliabo.infn.it
}

In May 2012 the CERN-CNGS neutrino beam operated in a dedicated short-bunched mode for two weeks in order to provide the opportunity to measure muon neutrino speed on an event-byevent basis. This new measurement campaign was the follow-up of a previous anomalous result reported by the OPERA Collaboration which was later revised and corrected by the Collaboration. The new analysis profited from the precision geodesy measurements of the neutrino baseline and of the CNGS/LNGS clock synchronization performed in the previous analysis. Different OPERA sub-detectors and timing systems were used and results were given separately for $v_{\mu}$ and $\bar{v}_{\mu}$ exploiting the information provided by the muon spectrometers.

36th International Conference on High Energy Physics

4-11 July 2012

Melbourne, Australia

${ }^{*}$ Speaker.

${ }^{\dagger}$ on behalf of the OPERA Collaboration. 


\section{Introduction}

In 2011 the OPERA experiment [1] at the Laboratori Nazionali del Gran Sasso (LNGS) reported the measurement of the time-of-flight (ToF) of muon neutrinos in the CNGS [2] beam. The ToF value obtained in 2011 [3] was shorter than what computed assuming the speed of light at the level of $2 \times 10^{-5}$. Later checks isolated two unaccounted systematic effects which completely absorbed the anomaly and provided a ToF value compatible with the speed of light [4]. Despite the robustness and reliability of the revised result the highly sensitive matter of the measurement demanded further checks with a new dedicated short-bunched neutrino beam. The new beam allowed the OPERA experiment to repeat the measurement with different techniques and with an improved timing system and also gave other LNGS experiments the opportunity to independently perform the measurement.

In the new run OPERA used scintillation detectors with an improved timing system and also exploited a dedicated independent timing system which served Resistive Plate Chamber (RPC) detectors inserted in the muon spectrometers. Finally outgoing muons from CC neutrino interactions were sign-tagged in order to provide ToF measurements separately for $v_{\mu}$ and $\bar{v}_{\mu}$.

\section{The new short-bunched neutrino beam}

In autumn 2011 a first CNGS short-bunch wide-spacing neutrino beam was used in order to cross-check the neutrino velocity result previously obtained by OPERA with a statistical method applied to the data accumulated with the standard CNGS neutrino beam tuned for the $v_{\tau}$ appearance search in $v_{\mu} \rightarrow v_{\tau}$ oscillations. This test proved that it was possible, within a short period of two to three weeks of bunched beam, to obtain similar or even better accuracy for the neutrino velocity measurement compared to the statistical analysis applied to the data accumulated with the standard CNGS beam over years.

The 2011 narrow-bunch beam had bunches separated by 524 ns yielding a total intensity of $1.1 \times 10^{12}$ protons per cycle. In the 2012 run with narrow-bunch narrow-spacing beam (BB) the bunch separation is only $100 \mathrm{~ns}$ in order to increase the number of delivered neutrinos per time unit. In each CNGS cycle, lasting $13.2 \mathrm{~s}$, a single extraction delivers 4 batches of 16 proton bunches. Each $1.8 \mathrm{~ns}$ long (RMS) bunch contains $10^{11}$ protons providing an intensity 6 times higher than during the $2011 \mathrm{BB}$ run.

Just before this running period CERN installed a monitoring system, called White Rabbit [5], at CERN and at LNGS to monitor relevant timing parameters of common systems and also specific parameters of each LNGS experiment. OPERA only used this facility to monitor the delay of the external GPS time signal along $8.3 \mathrm{~km}$ of the LNGS optical fiber and the frequency of the Master Clock (MC) oscillator which compromised the measurements performed in 2011.

\section{The detector and its timing systems}

An extensive description of the OPERA detector is given in [1]. Here we recall that the detector has a modular structure consisting of two instrumented target sections with a mass of about 625 tons followed by a magnetic muon spectrometer. Each target section is a sequence of walls filled 
with emulsion film/lead plate modules interleaved with pairs of horizontal and vertical $6.7 \times 6.7 \mathrm{~m}^{2}$ planes of 256 scintillator strips. Scintillators form the so called Target Tracker (TT) which allows the location of neutrino interactions in the target. Each of the two OPERA magnets is instrumented with 22 planes of RPC's. Each plane can provide the $x$ and $y$ transverse coordinates for crossing particles using horizontal and vertical readout strips.

Both TT and RPC sub-detector systems were used to measure the arrival time of neutrinos. The DAQ records the timestamp of the earliest TT photomultiplier signal of the event and the earliest hit of each RPC module reaching the readout electronics. A time calibration of these two sub-detectors allowed converting the measured time in UTC. The complete OPERA timing system is extensively described in [4] while Ref. [6] focuses on the improvements made for the new BB run. In particular a new dedicated timing system serving the RPC detectors was developed for this special run in order to improve the timing resolution and reduce the systematic error. It rests on the purposely developed electronics for triggering the Precision Tracker (PT) drift tubes: seven RPC layers in each magnet are instrumented with timing boards, each one discriminating the positive polarity signals and forming the OR from 16 read-out strips. Each RPC layer is served by 14 timing boards (TB), whose digital output signals are sent through $16 \mathrm{~m}$ long flat cables to the OR Plane Electronics (OPE) boards. A single-ended positive polarity output is present in the OPE and it was used for the new timing system. The UTC time of neutrino interactions with muons crossing the OPERA spectrometers is reconstructed in two steps: the UTC time coded in the synchronization signal (1PPmS), sent by the ESAT LNGS atomic clock, is acquired, while the sub-ms component is computed as a time difference between the event hits registered by the TB and the leading edge of the 1 PPmS measured by means of a TDC.

The decoding of the 1PPmS UTC time is performed by a custom VME module, called slave clock, which also reproduces with a negligible time jitter $(0.2 \mathrm{~ns})$ the $1 \mathrm{PPmS}$ signal in NIM format. The module output signals, seven for each spectrometer, are discriminated and also reshaped in NIM format. Signals from RPC's and the 1PPmS are converted to differential ECL and sent to a CAEN V767 VME TDC module The acquisition system is triggered by a 4/14 majority of the RPC layers. To obtain a ns-precision over the full range, the TDC was calibrated with a ppm precision. For this purpose a thermally stabilized oscillator producing a $5 \mathrm{kHz}$ reference signal sent into a TDC channel was used during the whole data taking period. This way, the stability of the TDC oscillator can be monitored event by event, performing also the calibration with the required precision.

\section{Data analysis and results}

Each event is classified according to its topology: CONTAINED are events with the neutrino vertex in the target sections; SPECTRO are events with the neutrino vertex in the spectrometer sections; FRONTMUON and SIDEMUON are events with the neutrino vertex outside the detector and with a muon entering from the upstream and lateral sides of the detector, respectively.

Independent analyses were performed for events involving TT and RPC detectors. Since $T O F_{c}$ (time of flight) is computed with respect to the origin of the OPERA reference frame which is located beneath the most upstream spectrometer magnet, the time measurements are corrected for 


\begin{tabular}{|l|c|c|c|c|c|}
\hline Event type & Events & Method 1 & Method 2 & Method 3 & Method 4 \\
\hline CONTAINED & 18 & 17 & 13 & 13 & 11 \\
FRONTMUON & 37 & 29 & 27 & 27 & 24 \\
SIDEMUON & 17 & 3 & 3 & 9 & 8 \\
SPECTRO & 20 & 10 & 5 & 9 & 6 \\
Others & 12 & 0 & 0 & 0 & 0 \\
\hline Total & 104 & 59 & 48 & 58 & 49 \\
\hline
\end{tabular}

Table 1: Events used by the four analysis methods.

the distance of the events along the beam line from this point, assuming time propagation at the speed of light.

Table 1 summarizes the total number of events recorded, as well as the number of events used in the four analysis methods described in the next subsections. Methods 1 and 2 were applied to TT data and Methods 3 and 4 to RPC data. Method 1 relies on the information of the earliest hit as described in [4]. Method 2 exploits the time information of all TT hits of the 3D muon track. The second method results in a better time resolution due to the larger amount of information associated with the whole track but misses NC-like events (Neutral Current). Method 3 reconstructs an independent mean time using RPC hits of the first and second Super Module separately and the same algorithm of Method 2. While Method 2 relies on standard DAQ Method 4

For events where the muon crosses at least one spectrometer its momentum and charge are measured. In 3 such events out of $58 \mathrm{CC}$ events used in this analysis, the muon charge is positive. One event is CONTAINED ( $p_{\mu} \simeq 12 \mathrm{GeV} / \mathrm{c}$ ), the other ones are FRONTMUON events $\left(p_{\mu} \simeq 46\right.$ $\mathrm{GeV} / \mathrm{c}$ and $p_{\mu} \simeq 79 \mathrm{GeV} / \mathrm{c}$ ). They exhibit a clear muon track crossing both spectrometers and thus all drift tube stations could be exploited for charge and momentum reconstruction. The overall charge mis-identification probability for these events is below $1.5 \%$. Considering also the charm background contribution, the number of expected fake positive muons from the whole BB sample is $<0.7$ for the 3 observed events. If the analysis is restricted only to contained events for which both charm contribution and charge mis-identification are lower, the expected number of fake positive muons is $<0.07$ for 1 observed event.

As discussed in the previous section, four different, correlated results were obtained for the neutrino velocity measurements using, respectively 1 . Standard DAQ, TT detectors and earliest hit timestamps, 2. Standard DAQ and TT hit timestamps of the reconstructed muon track, 3. Standard DAQ and RPC hit timestamps of the reconstructed muon track, 4. Timing Board system and RPC detectors using muon hit timestamps. Methods 1 and 2 are statistically correlated since they use the same DAQ system and sub-detector. They are also correlated with Method 3 using the same DAQ system. Method 4 is almost uncorrelated with the other methods because of the different timing system used. The residual correlation arises from the systematic error of the common part of the timing chain. The results obtained with each method are reported in Tab. 2, as well as the statistical and systematic errors. In order to obtain a single combined result, Methods 2 and 4 were used since they have the smallest statistical and systematic errors and - moreover - are almost completely uncorrelated thus providing maximum information. Values obtained for Method 1 were used for 


\begin{tabular}{|c|c|c|c|c|c|}
\hline Method & Events & $\delta t$ & Stat. & Syst. & Resol. \\
\hline 1 & 59 & -2.1 & 1.1 & 4.4 & 9.8 \\
2 & 48 & 1.2 & 1.0 & 3.3 & 6.5 \\
3 & 58 & -2.5 & 1.8 & 5.3 & 9.5 \\
4 & 49 & 0.6 & 0.4 & 3.6 & 1.1 \\
\hline
\end{tabular}

Table 2: Individual results for $v+\bar{v}$ for each of the four analysis methods reported in the text. In each row, the number of events, $\delta t \equiv T O F_{c}-T O F_{v}$ the statistical and systematic errors and the time resolution on single events are reported (not including the $1.8 \mathrm{~ns}$ RMS of the proton bunches). All time values are in ns.

events when Method 2 could not be used because of the absence of a muon track ( 20 events). A standard combination procedure in presence of correlated measurements was then used [7]. The final result is

$$
\delta t_{v}=0.7 \pm 0.4(\text { stat }) \pm 1.6(\text { syst. }- \text { uncorr. }) \pm 2.5(\text { syst. }- \text { corr. }) \text { ns. }
$$

Summing in quadrature the systematic errors, separately for $v$ and $\bar{v}$ contributions, leads to the results

$$
\left\{\begin{array}{l}
\delta t_{v}=0.6 \pm 0.4(\text { stat }) \pm 3.0(\text { syst }) \mathrm{ns} \\
\delta t_{\bar{v}}=1.7 \pm 1.4(\text { stat }) \pm 3.1(\text { syst. }) \mathrm{ns}
\end{array}\right.
$$

for neutrinos and anti-neutrinos respectively (the slight difference in the systematic errors arises from the different contributions of each method to them). When comparing $\delta t_{v}$ and $\delta t_{\bar{v}}$, systematic errors largely cancel out. It was assumed that all the NCÚlike events result from $v$ interactions. Since both results are compatible with zero, a limit on the deviation from the speed of light was derived (90\% C.L.):

$$
\left\{\begin{array}{l}
-1.8 \times 10^{-6}<\left(v_{v}-c\right) / c<2.3 \times 10^{-6} \\
-1.6 \times 10^{-6}<\left(v_{\bar{v}}-c\right) / c<3.0 \times 10^{-6}
\end{array}\right.
$$

for $v_{\mu}$ and $\bar{v}_{\mu}$ respectively. It is pointed out that the statistical errors in 4.2 were computed according to the RMS of the $v+\bar{v}$ distribution. The above results are in agreement with those obtained by the other LNGS experiments participating to this CNGS BB run [8]

\section{Conclusions}

In May 2012 a two-week dedicated CNGS proton beam was provided to perform a measurement of the neutrino velocity. The OPERA experiment after improving its timing system, has confirmed the result reported in [4], showing no significant deviation of the muon neutrino velocity from the speed of light. The present result is $\delta t_{v}=0.6 \pm 0.4($ stat $) \pm 3.0$ (syst.) ns, giving the limit $-1.8 \times 10^{-6}<\left(v_{v}-c\right) / c<2.3 \times 10^{-6}$ During the same period, 3 muon anti-neutrino interactions were also recorded. The corresponding $\delta t$ value is $1.7 \pm 1.4($ stat $) \pm 3.1$ (syst.) ns compatible with that obtained with neutrino events. 

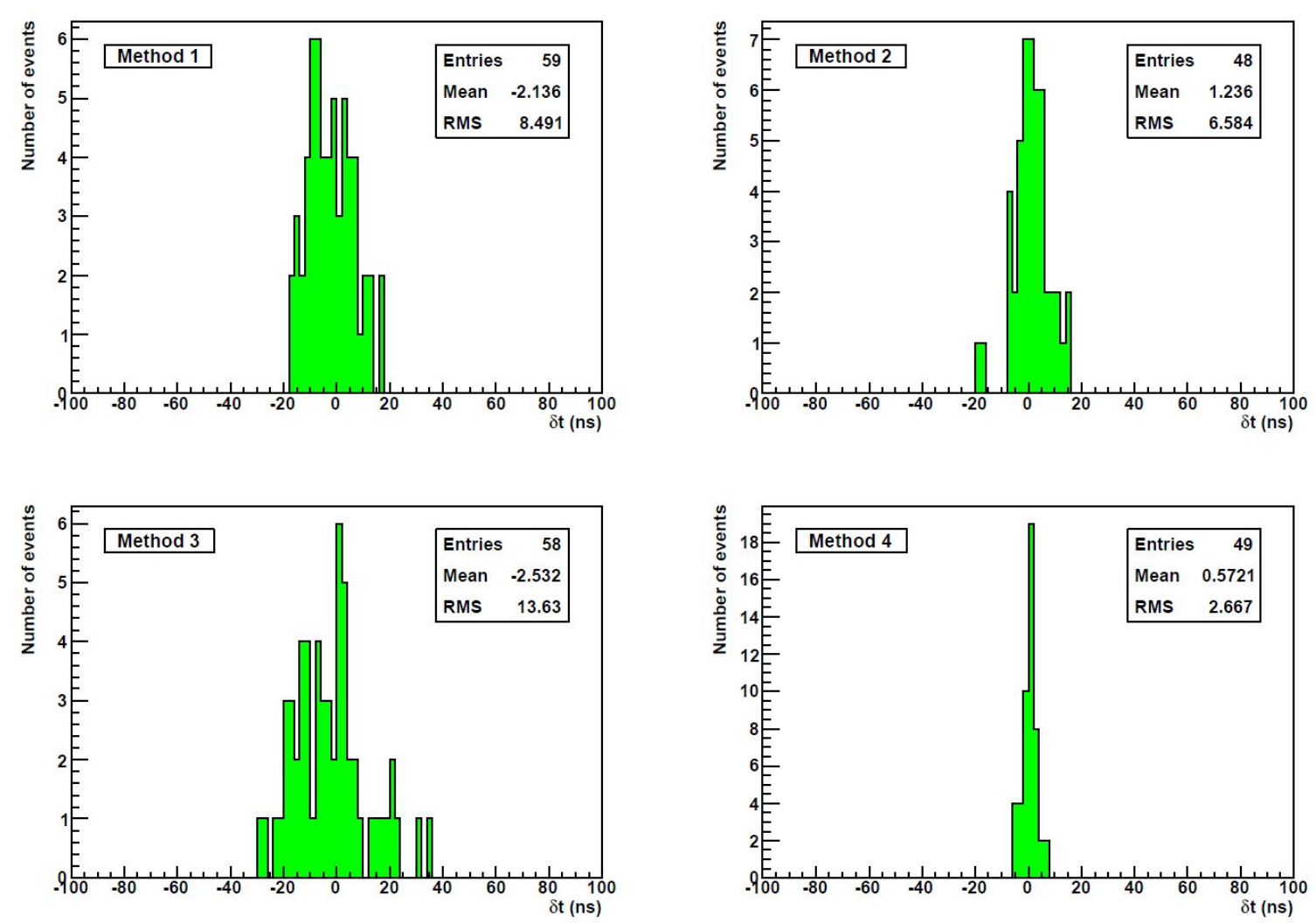

Figure 1: Neutrino ToF differences with respect to $c$ for the four analysis presented in this work.

\section{References}

[1] R. Acquafredda et al., The OPERA experiment in the CERN to Gran Sasso neutrino beam, JINST 4 (2009) P04018.

[2] G. Acquistapace et al., The CERN neutrino beam to Gran Sasso (NGS): Conceptual technical design, CERN-YELLOW-98-02.

[3] T. Adam et al., Measurement of the neutrino velocity with the OPERA detector in the CNGS beam, 2011, arXiv:1109.4897v2 [hep-ex].

[4] T. Adam et al., Measurement of the neutrino velocity with the OPERA detector in the CNGS beam, JHEP 1210 (2012) 093 [hep-ex/1109.4897v4].

[5] The White Rabbit system http://www. ohwr.org/projects/white-rabbit.

[6] T. Adam et al., Measurement of the neutrino velocity with the OPERA detector in the CNGS beam using the 2012 dedicated data, 2012, arXiv:1212.1276 [hep-ex].

[7] L. Lyons, D. Gibaut and P. Clifford, How to combine correlated estimates of a single physical quantity, Nucl. Instrum. Methods. A 270 (1988) 110.

[8] P. Alvarez Sanchez et al. [Borexino Collaboration], Measurement of CNGS muon neutrino speed with Borexino, Phys. Lett. B 716 (2012) 401; M. Antonello et al. [ICARUS Collaboration], Precision measurement of the neutrino velocity with the ICARUS detector in the CNGS beam, JHEP 1211 (2012) 049; N. Y. .Agafonova et al. [LVD Collaboration], Measurement of the velocity of neutrinos from the CNGS beam with the Large Volume Detector, Phys. Rev. Lett. 109 (2012) 070801. 